\title{
Transcranial color-coded duplex sonography assessment of cerebrovascular reactivity to carbon dioxide: an interventional study
}

\author{
Stephanie Klinzing ${ }^{1 *} \mathbb{D}$, Federica Stretti ${ }^{2}$, Alberto Pagnamenta ${ }^{3,4,5}$, Markus Bèchir ${ }^{1}$ and Giovanna Brandi ${ }^{1}$
}

\begin{abstract}
Background: The investigation of $\mathrm{CO}_{2}$ reactivity $\left(\mathrm{CO}_{2}-\mathrm{CVR}\right)$ is used in the setting of, e.g., traumatic brain injury (TBI). Transcranial color-coded duplex sonography (TCCD) is a promising bedside tool for monitoring cerebral hemodynamics. This study used TCCD to investigate $\mathrm{CO}_{2}$-CVR in volunteers, in sedated and mechanically ventilated patients without TBI and in sedated and mechanically ventilated patients in the acute phase after TBI.

Methods: This interventional investigation was performed between March 2013 and February 2016 at the surgical ICU of the University Hospital of Zurich. Ten volunteers (group 1), ten sedated and mechanically ventilated patients (group 2), and ten patients in the acute phase (12-36 h) after severe TBI (group 3) were included. $\mathrm{CO}_{2}$-CVR to moderate hyperventilation $\left(\triangle \mathrm{CO}_{2}-5.5 \mathrm{mmHg}\right.$ ) was assessed by TCCD.

Results: $\mathrm{CO}_{2}$-CVR was $2.14(1.20-2.70) \% / \mathrm{mmHg}$ in group 1, $2.03(0.15-3.98) \% / \mathrm{mmHg}$ in group 2, and 3.32 (1.184.48)\%/mmHg in group 3 , without significant differences among groups.

Conclusion: Our data did not yield evidence for altered $\mathrm{CO}_{2}$-CVR in the early phase after TBI examined by TCCD.

Trial registration: Part of this trial was performed as preparation for the interventional trial in TBI patients (clinicaltrials.gov NCT03822026, 30.01.2019, retrospectively registered).

Keywords: Transcranial color-coded duplex sonography, Intensive care ultrasound, CO2 reactivity, Traumatic brain injury, Cerebral blood flow measurements
\end{abstract}

\section{Background}

Cerebral autoregulation allows the maintenance of stable cerebral blood flow (CBF) despite changes in cerebral perfusion pressure (CPP) through variations of cerebral vascular resistance (CVR) [25]. Carbon dioxide $\left(\mathrm{CO}_{2}\right)$ is a potent cerebral vasodilator, with a sigmoid relationship between $\mathrm{paCO}_{2}$ (arterial carbon dioxide) and $\mathrm{CBF}$ that can be assumed to be linear during acute changes in normophysiologic states [7] and which is mediated by $\mathrm{CO}_{2}$ -related changes in extracellular $\mathrm{pH}$. This $\mathrm{CO}_{2}$ induced

\footnotetext{
*Correspondence: stephanie.klinzing@usz.ch

${ }^{2}$ Intensive Care Unit, Westmead Hospital, Westmead, NSW, Australia

Full list of author information is available at the end of the article
}

mechanism is commonly used in the clinical setting to reduce elevated intracranial pressure (ICP) by application of hyperventilation (HV), leading to hypocapnia. A decrease in $\mathrm{paCO}_{2}$ leads to a reduction in CBF, thus reducing cerebral blood volume, and, consequently, ICP. Changes in CVR and CBF in response to changes in $\mathrm{CO}_{2}$ are termed cerebrovascular reactivity to $\mathrm{CO}_{2}\left(\mathrm{CO}_{2}-\mathrm{CVR}\right)$.

Several invasive and non-invasive techniques are currently available to assess CBF. These include, e.g., arterial and jugular venous tracer-concentration measurements (Kety-Schmidt method), Xenon clearance technique, positron emission tomography, near-infrared spectroscopy (NIRS), and transcranial Doppler (TCD). The choice of technique is dependent on the clinical scenario. 
The non-invasive bedside ultrasonography technique of TCD is an attractive tool for determining CBF and $\mathrm{CO}_{2}$-CVR. Reference values for $\mathrm{CO}_{2}$-CVR assessed by TCD in healthy volunteers are reported to range between 2.9 and $3.7 \% / \mathrm{mmHg}[9,11,12,16,29]$. For patients under general anesthesia, however, the potential effect of anesthetic agents has to be taken into account. Current data suggest maintained CO2-CVR during anesthesia and generally accepted values of $2.5-6 \%$ change in $\mathrm{cm} / \mathrm{s} /$ mmHgCVR for $\mathrm{CO}_{2}$-CVR have been reported $[5,8,15$, $19,27,28]$. In TBI, cerebral circulation may be compromised after injury. Data suggest that $\mathrm{CO}_{2}-\mathrm{CVR}$ may be preserved or impaired at various stages of TBI $[12,14$, $21,24]$. Research concerning the association of impaired $\mathrm{CO}_{2}-\mathrm{CVR}$ and neurological outcome is ongoing, because conflicting results have been reported [3, 24].

Transcranial color-coded duplex sonography (TCCD) is an ultrasound technique, combining Doppler and Duplex effects, thus allowing the visualization of the examined vessels. As TCCD is more observer- independent than TCD [18], it could be an attractive tool for serial bedside measurements of flow velocities in the Intensive Care Unit (ICU) setting.

In the present interventional study, TCCD was used for assessing $\mathrm{CO}_{2}$-CVR. A systematic investigation of $\mathrm{CO}_{2}$-CVR by TCCD in healthy volunteers, patients on mechanical ventilation, and patients with TBI was conducted to investigate whether there is evidence for altered $\mathrm{CO}_{2}$-CVR in the acute phase of TBI in our study population.

\section{Methods}

This study was conducted as an interventional trial in the surgical ICU of the University Hospital of Zurich between March 2013 and February 2016. The Cantonal Ethics Committee of Zurich approved and registered the study (KEK-ZH 2012-0542). Informed written consent was obtained from all participants or next of kin prior to study enrollment and/or from the patient after ICU discharge.

Patients in the TBI group were included in a study focusing on the effect of moderate hyperventilation on cerebral metabolism and thus selected according to previously published inclusion criteria [2]. Part of this trial was performed as preparation for the interventional trial in TBI patients (clinicaltrials.gov NCT03822026, retrospectively registered).

\section{Patient population}

The study was conducted in spontaneously breathing volunteers (Group 1), sedated and mechanically ventilated patients with presumed preserved $\mathrm{CO}_{2}$-CVR (Group 2), and sedated and mechanically ventilated patients suffering from severe TBI (TBI Group 3).

Inclusion criteria for Group 3 were adults ( $\geq 18$ years of age) with non-penetrating head injury, with an initial Glasgow Coma Scale (GCS) score $<9$ prior to sedation and intubation, extended neuromonitoring with ICP, brain tissue oxygenation $\left(\mathrm{P}_{\mathrm{br}} \mathrm{O}_{2}\right)$, and/or microdialysis probes (TBI group), and also undergoing invasive mechanical ventilation with $\mathrm{FIO}_{2}<60 \%$ and PEEP $<15$ $\mathrm{cmH}_{2} \mathrm{O}$. Exclusion criteria for all groups were decompressive craniectomy, pregnancy, pre-existing neurologic disease, previous TBI, acute cardiovascular disease, severe respiratory failure, acute or chronic liver disease, sepsis, and failure to obtain satisfactory bilateral TCCD signals. Patients with persisting hypovolemia or hemodynamic instability despite previous fluid resuscitation (defined as Global End-Diastolic Volume Index $<680 \mathrm{ml} /$ $\mathrm{m}^{2}$, central venous oxygen saturation $\left(\mathrm{ScvO}_{2}\right)<60 \%$ and/ or increase in mean arterial blood pressure (MAP) $>15 \%$ after passive leg raising test) were excluded.

The study was performed in the acute phase (12-36 h) after severe TBI (Group 3), while patients in Group 2 were investigated within $36 \mathrm{~h}$ after onset of mechanical ventilation.

All TBI patients were treated according to a cerebral perfusion orientated protocol aiming to achieve $\mathrm{CPP}>70 \mathrm{mmHg}, \mathrm{ICP} \leq 20 \mathrm{mmHg}, \mathrm{P}_{\text {br }} \mathrm{O}_{2}>15 \mathrm{mmHg}$, $\mathrm{P}_{\mathrm{a}} \mathrm{CO}_{2}$ between 4.8 and $5.2 \mathrm{kPa}$. For Group 2, a MAP of $65 \mathrm{mmHg}$ was targeted.

\section{TCCD measurements}

TCCD examination of the middle cerebral artery (MCA) was performed bilaterally via the transtemporal acoustic window by two experienced investigators (GB, SK), following standard techniques using a 5-1 $\mathrm{MHz}$ Probe (Philips CX 50, USA) [17]. Three repeated measurements of the peak systolic (PSV) and end-diastolic (EDV) velocity were performed for each side and an average value was calculated. The device also automatically calculated CBF-velocity (CBFV) and pulsatility index (PI).

\section{Study protocol}

In Group 1, ten spontaneously breathing volunteers were examined (Fig. 1, Panel A) using end-tidal carbon dioxide $\left(\mathrm{EtCO}_{2}\right)$ to monitor ventilation. Subsequently, each volunteer was asked to gradually increase respiratory rate and tidal volume to achieve a reduction in $\mathrm{EtCO}_{2}$ of approximatively $5.5 \mathrm{mmHg}$. Once the desired $\triangle \mathrm{ETCO}_{2}$ was achieved, the volunteer maintained a stable minute ventilation and $\mathrm{EtCO}_{2}$ for the duration of the TCCD measurements. After the TCCD measurements, the volunteer returned to resting ventilation. 


\section{Study protocol}

Panel A

\section{A}

B

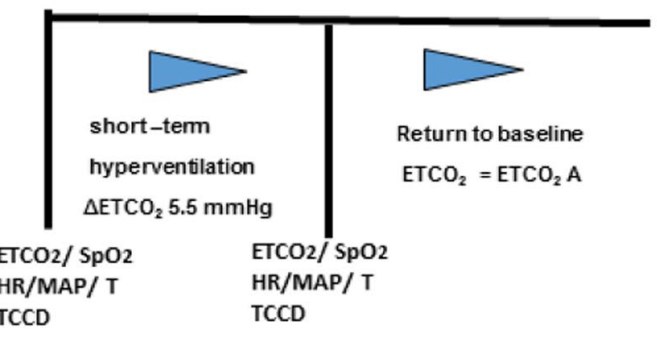

Panel B
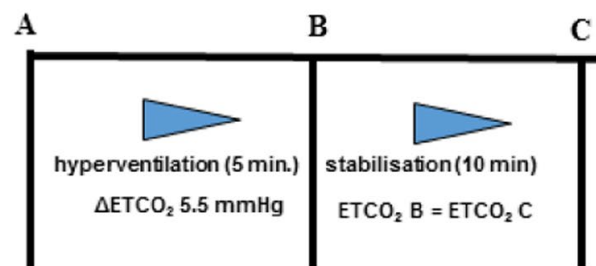

B

C

D
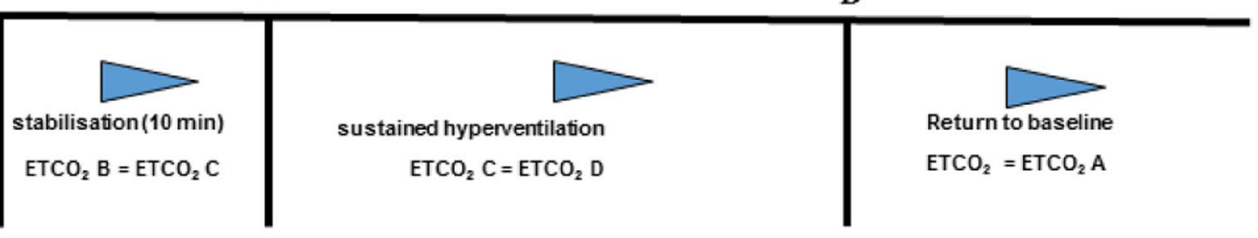

$\mathrm{ETCO} 2 / \mathrm{MV} / \mathrm{SpO} 2$ HR/MAP/ICP/CPP ABGA

$\mathrm{ETCO} 2 / \mathrm{MV} / \mathrm{SpO} 2$ HR/MAP/ICP/CPP

ETCO2/ MV/SpO2
HR/MAP/ICP/CPP
ABGA

$\mathrm{ETCO} 2 / \mathrm{MV} / \mathrm{SpO} 2$ HR/MAP/ICP/CPP ABGA

TCCD

Fig. 1 Study protocol. Panel A. Study protocol for group 1. During baseline conditions (A) and after short-term hyperventilation (B) the following parameters were recorded: end-tidal $\mathrm{CO}_{2}\left(\mathrm{ETCO}_{2}\right)$, peripheral capillary oxygen saturation $\left(\mathrm{SpO}_{2}\right)$, heart rate $(\mathrm{HR})$, mean arterial pressure (MAP), and auricular temperature (T). Measurements with transcranial color-coded duplex sonography (TCCD) were performed at time points $\mathbf{A}$ and $\mathbf{B}$. Panel B. Study protocol for groups 2 and 3. During baseline conditions (A), after short-term hyperventilation (B), stabilization (C), and sustained hyperventilation (D) several parameters were recorded. $\mathrm{ETCO}_{2}$ remained stable during $\mathbf{B}, \mathbf{C}$, and $\mathbf{D}$. TCCD measurements were performed during $\mathbf{A}$, $\mathbf{C}$, and $\mathbf{D}$. An arterial blood gas analysis (ABGA) was obtained during $\mathbf{A}, \mathbf{C}$, and $\mathbf{D}$. Only for patients in group 3 were values for intracranial pressure (ICP) and cerebral perfusion pressure (CPP) noted during A, B, C, and $\mathbf{D}$. MV: minute volume

Ten sedated and mechanically ventilated ICU patients in Group 2 and ten patients with severe TBI in Group 3 were investigated (Fig. 1, Panel B).

Under baseline conditions, a TCCD examination was performed and all variables were recorded (Fig. 1, point $\mathrm{A})$. The minute ventilation was then increased over a 10-min period to obtain moderate HV by a stepwise increase in tidal volume and respiratory rate until a reduction of $\mathrm{EtCO}_{2}$ of $0.7 \mathrm{kPa}$ (Fig. 1, point B) was achieved.

After 10 min of stable $\mathrm{EtCO}_{2}$, a second TCCD measurement was undertaken (begin of $\mathrm{HV}$, Fig. 1, point $\mathrm{C}$ ). The $\mathrm{EtCO}_{2}$ value was kept stable for $40 \mathrm{~min}$, and then followed by a third TCCD examination (Fig. 1, point D). Finally, normoventilation was re-established over $10 \mathrm{~min}$ and all variables were allowed to return to baseline (Fig. 1, point E). A final TCCD examination was conducted at this time point. At each time point, $\mathrm{MAP}, \mathrm{SpO}_{2}$ and $\mathrm{EtCO}_{2}$ were recorded.
Arterial blood gas tests (ABG) were obtained at points $\mathrm{A}, \mathrm{C}, \mathrm{D}$ and $\mathrm{E}$, to monitor the changes in $\mathrm{pH}$ and $\mathrm{P}_{\mathrm{a}} \mathrm{CO}_{2}$.

For study purpose, measurements and values obtained at timepoint $\mathrm{A}$ and $\mathrm{B}$ was used for group 1, while timepoint $A$ and $D$ was used for group 2 and 3.

\section{Definition of cerebrovascular reactivity to carbon dioxide} $\mathrm{CO}_{2}$-CVR is expressed in terms of absolute and relative reactivity. Absolute $\mathrm{CO}_{2}$-CVR is defined as change in $\mathrm{MFV}(\mathrm{cm} / \mathrm{s})$ per $\mathrm{mmHg}$ change in $\mathrm{CO}_{2}$. Relative $\mathrm{CO}_{2}$-CVR is defined as percentage change compared to baseline value.

$$
\text { Absolute } \mathrm{CO}_{2}-\mathrm{CVR}=\Delta \mathrm{MFV} / \Delta \mathrm{CO}_{2}
$$

Relative $\mathrm{CO}_{2}-\mathrm{CVR}=\left(\right.$ Absolute $\mathrm{CO}_{2}-\mathrm{CVR} /$ baseline MFV $) \times 100$ 
As the relative reactivity is less dependent on baseline values, it has been proposed as a more valuable indicator of $\mathrm{CO}_{2}$-CVR for analysis [10]. Relative reactivity was therefore chosen as the indicator for $\mathrm{CO}_{2}-\mathrm{CVR}$.

$\triangle \mathrm{MFV}=$ difference in MFV between baseline and after $\mathrm{HV}$.

$\Delta \mathrm{CO}_{2}=$ difference in $\mathrm{CO}_{2}$ between baseline and after $\mathrm{HV}$. In Group 1, EtCO 2 was used, while $\mathrm{PaCO}_{2}$ was used in Group 2 and TBI Group 3.

Hyperventilation constricts distal vessels, so a decrease in the absolute value of MFV is expected is the major intracranial vessels, as the ones investigated by TCCD.

\section{Statistical analysis}

Descriptive statistics were presented as mean with standard deviation (SD) or as median with interquartile range (IQR) for quantitative data. Categorical data were presented as absolute numbers with percentages. Comparisons of continuous variables among the three groups were performed with one-way analysis of variance or with the Kruskal-Wallis-test, as appropriate. For statistically significant $p$-values, post-hoc tests were performed, taking the multiple comparisons into account. Qualitative data among the three groups were compared with the Chi-Square test. In cases of statistically significant results, post-hoc comparisons were made with the appropriate critical level adjustment. Comparisons of quantitative data before and during hyperventilation were conducted with the paired Student's $t$-test or with the Wilcoxon matched pairs test, as appropriate. All tests were done two-sided, and $p$-values $<0.05$ were considered statistically significant. Stata version 12.1 (StatCorp. LP, College Station, TX, USA) was used for all statistical analysis.

\section{Results}

Baseline characteristics of Group 1, Group 2 and Group 3 are presented in Table 1 . As stated in exclusion criteria, patients and volunteers included did not have comorbidities with known impact on cerebral autoregulation. Patients included in group 2 were admitted to the ICU after surgical care (Otolaryngoly $(n=3)$, plastic surgery $(n=2)$, thoracic surgery $(n=2)$, visceral $\operatorname{surgery}(n=3))$. Patients in Group 3 were under higher dosages of midazolam $(p<0.001)$, propofol $(p=0.004)$, fentanyl $(p=0.02)$, and norepinephrine $(p=0.008)$ compared to Group 2, while groups were comparable according to age, sex and BMI.

All patients included to group 3 showed traumatic subarachnoidal hemorrhage on the initial CT scan. Seven patients showed bilateral contusional hemorrhage and three patients predominantly left sided contusional hemorrhage. Seven Patients were classified as Marshall 2, one

Table 1 Baseline characteristics

\begin{tabular}{|c|c|c|c|c|}
\hline Parameter & $\begin{array}{l}\text { Group } 1 \\
n=10\end{array}$ & $\begin{array}{l}\text { Group } 2 \\
n=10\end{array}$ & $\begin{array}{l}\text { Group } 3 \\
n=10\end{array}$ & $p$ \\
\hline Age (years) & $34 \pm 7.5$ & $44 \pm 16.3$ & $35 \pm 13.8$ & 0.21 \\
\hline Sex (men) & 8 & 4 & 7 & 0.16 \\
\hline $\mathrm{BMI}$ & $25.4 \pm 3.7$ & $23.1 \pm 2.5$ & $24.0 \pm 2.7$ & 0.25 \\
\hline SAPS II & n.a & $27 \pm 11$ & $47 \pm 8$ & $<0.001$ \\
\hline GCS & n.a & n.a & $5.9 \pm 2.8$ & n.a \\
\hline ISS & n.a & n.a & $30 \pm 11$ & n.a \\
\hline \multicolumn{5}{|l|}{ Depth of insonation MCA (mm) } \\
\hline Right & $47 \pm 5$ & $52 \pm 4$ & $54 \pm 5$ & $0.03^{\#}$ \\
\hline Left & $48 \pm 4$ & $51 \pm 6$ & $52 \pm 5$ & 0.10 \\
\hline \multicolumn{5}{|l|}{ Angle of insonation $\left({ }^{\circ}\right)$} \\
\hline Right & $22 \pm 10$ & $36 \pm 8$ & $28 \pm 12$ & $0.01^{*}$ \\
\hline Left & $25 \pm 14$ & $33 \pm 13$ & $25 \pm 8$ & 0.26 \\
\hline Midazolam (mg/kg/h) (n) & n.a & $0.0 \pm 0.0(0)$ & $0.3 \pm 0.3(7)$ & 0.002 \\
\hline Propofol (mg/kg/h) (n) & n.a & $4.3 \pm 1.0(10)$ & $2.0 \pm 1.8(6)$ & 0.003 \\
\hline Remifentanil (mg/kg/h) (n) & n.a & $2.3 \pm 2.3(6)$ & $1.5 \pm 2.2(4)$ & 0.4 \\
\hline Fentanyl (mg/kg/h) (n) & n.a & $0.1 \pm 0.4(3)$ & $2.8 \pm 3.0(6)$ & 0.02 \\
\hline Norepinephrine ( $\mu \mathrm{cg} / \mathrm{kg} / \mathrm{min})(\mathrm{n})$ & n.a & $0.10 \pm 0.06(9)$ & $0.28 \pm 0.17(9)$ & 0.004 \\
\hline
\end{tabular}

Values are expressed as mean (standard deviation) or $\mathrm{n}$ as appropriate

n.a. not applicable, BMI body mass index, SAPS // simplified acute physiology score II, GCS Glasgow Coma scale; ISS- Injury Severity Score; MCA: middle cerebral artery

* between Group 1 and 2. " between Group 1 and 3 
patient as Marshall 3, one patient as Marshall 5 and two patients as Marshall 6.

While HR remained stable in all groups, MAP was significantly different between Group 1 and Group 2 $(p=0.001$ and $p=0.008)$ and between Group 2 and Group $3(p=0.001$ and $p=0.005)$ at baseline and during $\mathrm{HV}$. HV lead to a significant increase in $\mathrm{MV}$ and corresponding decrease in $\mathrm{EtCO}_{2}$ and $\mathrm{P}_{\mathrm{a}} \mathrm{CO}_{2}$ as well as a significant reduction of MFV in the right and left MCA in all groups (Table 2). Baseline MFV did not differ significantly between group 2 and 3, but was significantly higher at baseline in group 3 compared to group 1 ( $p=0.024$ (right), $p=0.032$ (left)).

Absolute and relative values for $\mathrm{CO}_{2}-\mathrm{CVR}$ for all groups are presented in Table $3 . \mathrm{CO}_{2}$-CVR was $2.14(1.20-2.70)$ $\% / \mathrm{mmHg}$ in group $1,2.03(0.15-3.98) \% / \mathrm{mmHg}$ in group 2 , and $3.32(1.18-4.48) \% / \mathrm{mmHg}$ in group 3.

Neither the $\mathrm{CO}_{2}$-CVR within-groups (comparison of the more- with the less-injured side) nor between-groups were significantly different.

\section{Discussion}

\section{Main findings}

The present study used TCCD to assess $\mathrm{CO}_{2}$-CVR in healthy volunteers, patients under sedation and mechanical ventilation without TBI and patients with severe TBI in the first 12-36 h after trauma. TCCD was conducted in the acute phase after TBI as part of another study. [2]

A relative $\mathrm{CO}_{2}$ - CVR of $2.14 \% / \mathrm{mmHg}$ (95\% CI 1.20 2.70) was found in volunteers, $2.03 \% / \mathrm{mmHg}$ ( $95 \%$ CI $0.15-3.98)$ in sedated and mechanically ventilated patients and $3.32 \% / \mathrm{mmHg}(95 \% \mathrm{CI} 1.18-4.48)$ in patients
Table 3 Cerebrovascular carbon dioxide reactivity

\begin{tabular}{ccccc}
\hline Characteristic & Group 1 & Group 2 & Group 3 & p \\
\hline Absolut CVR-CO & $((\mathrm{cm} / \mathrm{s}) / \mathrm{mmHg})$ & & \\
right & $1.16(0.76-$ & $1.49(0.15-$ & $2.08(1.22-$ & 0.14 \\
& $1.78)$ & $3.26)$ & $3.49)$ & \\
left & $1.12(0.52-$ & $0.93(-0.2-4.00)$ & $2.12(0.36-$ & 0.38 \\
& $1.58)$ & & $4.77)$ & \\
overall & $1.11(0.71-$ & $1.52(0.02-$ & $2.36(0.80-$ & 0.17 \\
& $1.58)$ & $3.21)$ & $4.34)$ & \\
Relative $\mathrm{CVR}^{-C_{2}}(\% / \mathrm{mmHg})$ & & & \\
right & $2.25(1.36-$ & $2.07(0.45-$ & $2.64(1.76-$ & 0.4 \\
& $2.78)$ & $3.93)$ & $4.51)$ & \\
left & $2.15(0.84-$ & $1.49(-0.53-4.5)$ & $2.88(0.51-$ & 0.4 \\
& $2.95)$ & & $5.48)$ & \\
overall & $2.14(1.20-$ & $2.03(0.15-$ & $3.32(1.18-$ & 0.28 \\
& $2.70)$ & $3.98)$ & $4.48)$ & \\
\hline
\end{tabular}

Values are expressed as median $(95 \% \mathrm{Cl})$

$\mathrm{CVR}-\mathrm{CO}_{2}$ cerebrovascular carbon dioxide reactivity

in the acute phase after TBI. $\mathrm{CO}_{2}$-CVR values between groups was not significantly different.

\section{How our data compare to the literature}

In our TCCD study, relative $\mathrm{CO}_{2}$-CVR values in healthy volunteers $2.14 \% / \mathrm{mmHg}$ (95\% CI $1.20-2.70$ ) were lower than those obtained by Klingelhofer et al.[12], which showed a mean $\mathrm{CO}_{2}$-CVR of $3.7 \pm 0.5 \% / \mathrm{mmHg}$. Flow velocities obtained via TCCD might be higher than TCD values due to correction of the angle of incidence in TCCD measurements [1]. This may influence relative $\mathrm{CO}_{2}$-CVR when TCCD is used. For patients under

Table 2 Physiological data

\begin{tabular}{|c|c|c|c|c|c|c|c|c|c|}
\hline \multirow[b]{2}{*}{ Parameter } & \multicolumn{3}{|l|}{ Group 1} & \multicolumn{3}{|l|}{ Group 2} & \multicolumn{3}{|l|}{ Group 3} \\
\hline & Baseline & $\begin{array}{l}\text { Short-term } \\
\text { Hyperventilation }\end{array}$ & $\mathbf{p}$ & Baseline & $\begin{array}{l}\text { Sustained } \\
\text { Hyperventilation }\end{array}$ & $\mathbf{p}$ & Baseline & $\begin{array}{l}\text { Sustained } \\
\text { Hyperventilation }\end{array}$ & $\mathbf{p}$ \\
\hline $\mathrm{HR}(\mathrm{b} / \mathrm{min})$ & $77 \pm 14$ & $78 \pm 14$ & 0.41 & $74 \pm 18$ & $75 \pm 21$ & 0.84 & $73 \pm 17$ & $72 \pm 18$ & 0.61 \\
\hline MAP (mmHg) & $93 \pm 8^{1}$ & $93 \pm 8^{2}$ & 0.94 & $76 \pm 11^{3}$ & $78 \pm 12^{4}$ & 0.62 & $93 \pm 9$ & $94 \pm 11$ & 0.60 \\
\hline MV (I/min) & n.a & n.a & - & $6.4 \pm 1.7$ & $9.2 \pm 2.7$ & $<0.001$ & $7.1 \pm 1.4$ & $8.9 \pm 1.7$ & 0.0037 \\
\hline $\mathrm{ETCO}_{2}(\mathrm{mmHg})$ & $38.6 \pm 3.7$ & $30.8 \pm 2.4$ & $<0.001$ & $41.4 \pm 6.9$ & $34.4 \pm 7.1$ & $<0.001$ & $37.5 \pm 5.5$ & $31.8 \pm 4.8$ & $<0.001$ \\
\hline $\mathrm{pH}$ & n.a & n.a & - & $7.36 \pm 0.05$ & $7.42 \pm 0.05$ & $<0.001$ & $7.37 \pm 0.09$ & $7.45 \pm 0.02$ & $<0.001$ \\
\hline $\mathrm{paCO}_{2}(\mathrm{mmHg})$ & $38.5 \pm 3.6^{*}$ & $30.6 \pm 2.4^{*}$ & $<0.001$ & $39.3 \pm 4.6$ & $33.3 \pm 4.4$ & $<0.001$ & $37.4 \pm 5.5$ & $31.8 \pm 4.7$ & $<0.001$ \\
\hline \multicolumn{10}{|l|}{ MFV MCA $(\mathrm{cm} / \mathrm{s})$} \\
\hline Right & $55 \pm 10^{5}$ & $45 \pm 7$ & 0.0004 & $63 \pm 25$ & $51 \pm 18$ & 0.03 & $78 \pm 22$ & $65 \pm 18$ & $<0.001$ \\
\hline Left & $55 \pm 11^{6}$ & $47 \pm 9$ & 0.0003 & $65 \pm 21$ & $56 \pm 14$ & 0.04 & $78 \pm 22$ & $62 \pm 12$ & 0.005 \\
\hline
\end{tabular}

Values are expressed as mean \pm standard deviation

$H R$ heart rate, $M A P$ mean arterial pressure, $M V$ minute ventilation, $E T C O_{2}$ end-tidal carbon dioxide, $M F V$ mean flow velocity, $M C A$ middle cerebral artery, n.a. not applicable

* Assumption of $\mathrm{ETCO}_{2}=\mathrm{paCO}_{2}$

${ }^{1} p=0.001$ between group 1 and $2 ;{ }^{2} p=0.008$ between group 1 and $2 ;{ }^{3} p=0.001$ between group 2 and $3 ;{ }^{4} p=0.005$ between group 2 and $3 ;{ }^{5} p=0.024$ between group 1 and $3 ;{ }^{6} p=0.032$ between group 1 and 3 
general anesthesia undergoing major surgery, $\mathrm{CO}_{2}-\mathrm{CVR}$ assessed with TCD was reported to be preserved and mainly comparable with that of healthy volunteers $[5$, $8,19,27,28]$. This suggests a negligible influence of routinely used anesthetic agents on $\mathrm{CO}_{2}$-CVR. In our study, patients received intravenous analgosedation with Propofol and Remifentanil or Fentanil, in accordance to the referred studies, we did not find evidence of impact of those agents on $\mathrm{CO}_{2}-\mathrm{CVR}$. Current values of CO2-CVR around $2.5-6 \%$ change in $\mathrm{cm} / \mathrm{s} / \mathrm{mmHg}$ are generally accepted [15]. In accordance with published data, we found a preserved $\mathrm{CO}_{2}-\mathrm{CVR}$ in our group of sedated and mechanically ventilated patients without TBI $[5,8,19,27,28]$.

In our TBI patients, $\mathrm{CO}_{2}$-CVR was $3.32 \% / \mathrm{mmHg}(95 \%$ CI 1.18-4.48). However, the increase in $\mathrm{CO}_{2}$-CVR did not reach statistical significance. Comparing our data with that in existing literature, some aspects deserve consideration. Klingelhofer et al. [12] reported a decreased but preserved $\mathrm{CO}_{2}$-CVR of $2.0 \pm 1.1 \% / \mathrm{mmHg}$ in 40 patients with acute traumatic and spontaneous cerebral hemorrhage, of whom 24 were in barbiturate coma. As barbiturates have been shown to influence $\mathrm{CO}_{2}$-CVR by metabolic suppression [23], this needs to be taken into account. $\mathrm{CO}_{2}$-CVR was reported to be preserved in other studies with TBI patients, although especially in the acute phase after TBI, impaired $\mathrm{CO}_{2}$-CVR was observed $[14,21,24,26]$.

In comparison with the cumbersome direct measurement of CBF, the non-invasive, bedside tool of sonography has the advantage of serial measurements of MFV and $\mathrm{CO}_{2}$-CVR in critically ill patients, although invasive and non-invasive methods complement each other, depending on the clinical scenario.

In our opinion, TCCD offers advantages compared to TCD in the daily setting of an ICU for non-continuous serial measurements, as it has been proven to be less operator dependent [18]. Furthermore, good reliability of interobserver results of TCCD measurements in TBI patients for trained operators has been reported, thus underscoring the value of TCCD to obtain reliable measurements [4]. This is an important aspect in the ICU setting, where serial measurements are performed by variably skilled operators. We were previously able to demonstrate a steep learning curve for residents introduced to TCCD in healthy volunteers [13]. Depending on the clinical scenario, TCCD seems to be interchangeable with TCD for serial monitoring of $\mathrm{CO}_{2}$-CVR, while TCD offers the advantage of continuous monitoring over time with a fixed probe.

TBI patients have been shown to have impaired cerebrovascular reactivity during long periods of their ICU stay, with a limited impact of current ICU treatment and an association of impaired cerebrovascular reactivity and outcome [6,30]. Our study results do not suggest impaired $\mathrm{CO}_{2}-\mathrm{CVR}$. Of notice, $\mathrm{CO}_{2}-\mathrm{CVR}$ is only one of several mechanism of cerebral autoregulation, thus preserved $\mathrm{CO}_{2}$-CVR does not imply intact cerebral autoregulation. While on the one hand it is known that prolonged HV can negatively affect outcome[20], on the other hand it has been postulated that hyperventilation, when CO2-CVR is intact, temporarily improves cerebral autoregulation[22]. Thus, our finding of preserved CO2-CVR in the early phase after TBI encourages that cautious hyperventilation under monitoring may be considered a therapeutic option [2]. Furthermore, TCCD may serve as a monitoring tool for serial assessment of $\mathrm{CO} 2-\mathrm{CVR}$, which may change during the course of TBI, to detect signs of deterioration or recovery of CO2-CVR.

\section{Limitations}

One limitation of this study is the small sample size; our results should be confirmed in larger studies of TBI patients. As well, the number of volunteers and patients examined in our number is too small to establish reference values. In a larger study, TCCD measurements for the assessment of CO2-CVR should be performed taking the localization of the insult into account. Furthermore, TCCD measurements for the assessment of $\mathrm{CO}_{2}-\mathrm{CVR}$ should be performed in both the early and later time course after trauma, taking the localization of the insult into account. Finally, a comparison of $\mathrm{CO}_{2}$-CVR obtained by TCCD and TCD would be desirable.

\section{Conclusion}

Our data did not yield evidence for altered $\mathrm{CO}_{2}$-CVR in the early phase after TBI and TCCD a reliable tool for determination of $\mathrm{CO}_{2}$-CVR.

\section{Abbreviations \\ ABGA: Arterial blood gas analysis; $\mathrm{BMI}$ : Body mass index; $\mathrm{CO}_{2}-\mathrm{CVR}: \mathrm{CO}_{2}$ reactiv- ity; CPP: Cerebral perfusion pressure; $\mathrm{ETCO}_{2}$ : End-tidal carbon dioxide; $\mathrm{HR}$ : Heart rate; HV: Hyperventilation; ICU: Intensive care unit; MAP: Mean arterial pressure; MCA: Middle cerebral artery; MFV: Mean flow velocity; MV: Minute ventilation; SAPS II: Simplified acute physiology score II;TBI:Traumatic brain injury; TCD: Transcranial doppler sonography; TCCD: Transcranial color-coded duplex sonography.}

\section{Acknowledgements}

We thank all volunteers for their highly-appreciated participation in this study.

\section{Authors' contributions}

SK and GB designed and performed the study, collected data and drafted the paper. FS collected and interpreted data and critically revised a draft version. AP analysed and interpreted data, also carrying out a critical revision of the draft. MB contributed substantial intellectual input to the design and performance of the study as well as checking interpretation of data and undertaking a critical revision of the draft. All authors read and approved the manuscript.

Funding

None. 


\section{Availability of data and materials}

The datasets used and analyses during the current study are available from the corresponding author on reasonable request.

\section{Declarations}

\section{Ethics approval and consent to participate}

All procedures performed in studies involving human participants were in accordance with the ethical standards of the institutional research committee (Kantonale Ethikkommission Zürich, Switzerland) and with the 1964 Helsinki declaration and its later amendments or comparable ethical standards. The Cantonal Ethics Committee of Zurich approved and registered the study (KEKZH 2012-0542).Informed written consent was obtained from all participants or next of kin prior to study enrollment and/or from the patient after ICU discharge.

\section{Consent for publication}

Not applicable.

\section{Competing interests}

The authors declare that they have no competing interests.

\section{Author details}

${ }^{1}$ Institute for Intensive Medicine, University Hospital of Zurich, Raemistrasse 100, CH-8091 Zurich, Switzerland. ${ }^{2}$ Intensive Care Unit, Westmead Hospital, Westmead, NSW, Australia. ${ }^{3}$ Intensive Care Unit, Regional Hospital of Mendrisio, Mendrisio, Switzerland. ${ }^{4}$ Unit of Clinical Epidemiology, Ente Ospedaliero Cantonale, Bellinzona, Switzerland. ${ }^{5}$ Division of Pneumology, University of Geneva, Geneva, Switzerland.

Received: 24 April 2020 Accepted: 6 July 2021

Published online: 07 August 2021

\section{References}

1. Bartels E. Transcranial color-coded duplex ultrasound-possibilities and limits of this method in comparison with conventional transcranial Doppler ultrasound. Ultraschall Med. 1993;14:272-8.

2. Brandi G, Stocchetti N, Pagnamenta A, et al. Cerebral metabolism is not affected by moderate hyperventilation in patients with traumatic brain injury. Crit Care. 2019;23:45

3. Carmona Suazo JA, Maas Al, Van Den Brink WA, et al. CO2 reactivity and brain oxygen pressure monitoring in severe head injury. Crit Care Med. 2000;28:3268-74.

4. Dupont G, Burnol L, Jospe R, et al. Transcranial Color Duplex Ultrasound: A Reliable Tool for Cerebral Hemodynamic Assessment in Brain Injuries. J Neurosurg Anesthesiol. 2016;28:159-63.

5. Eng C, Lam AM, Mayberg TS, et al. The influence of propofol with and without nitrous oxide on cerebral blood flow velocity and $\mathrm{CO} 2$ reactivity in humans. Anesthesiology. 1992;77:872-9.

6. Froese L, Batson C, Gomez A, et al. The limited impact of current therapeutic interventions on cerebrovascular reactivity in traumatic brain injury: a narrative overview. Neurocrit Care. 2021;34:325-35.

7. Grubb RL Jr, Raichle ME, Eichling JO, et al. The effects of changes in PaCO2 on cerebral blood volume, blood flow, and vascular mean transit time. Stroke. 1974:5:630-9.

8. Harrison JM, Girling KJ, Mahajan RP. Effects of target-controlled infusion of propofol on the transient hyperaemic response and carbon dioxide reactivity in the middle cerebral artery. Br J Anaesth. 1999;83:839-44.

9. Izumi Y, Tsuda Y, Ichihara S, et al. Effects of defibrination on hemorheology, cerebral blood flow velocity, and $\mathrm{CO} 2$ reactivity during hypocapnia in normal subjects. Stroke. 1996;27:1328-32.

10. Kaiser L. Adjusting for baseline: change or percentage change? Stat Med. 1989;8:1183-90.

11. Kastrup A, Thomas C, Hartmann C, et al. Sex dependency of cerebrovascular CO2 reactivity in normal subjects. Stroke. 1997;28:2353-6.

12. Klingelhofer J, Sander D. Doppler CO2 test as an indicator of cerebral vasoreactivity and prognosis in severe intracranial hemorrhages. Stroke. 1992:23:962-6.
13. Klinzing S, Steiger P, Schupbach RA, et al. Competence for transcranial color-coded Duplex sonography is rapidly acquired. Minerva Anestesiol. 2015:81:298-304

14. Lee JH, Kelly DF, Oertel $M$, et al. Carbon dioxide reactivity, pressure autoregulation, and metabolic suppression reactivity after head injury: a transcranial Doppler study. J Neurosurg. 2001;95:222-32.

15. Mariappan R, Mehta J, Chui J, et al. Cerebrovascular reactivity to carbon dioxide under anesthesia: a qualitative systematic review. J Neurosurg Anesthesiol. 2015;27:123-35.

16. Markwalder TM, Grolimund P, Seiler RW, et al. Dependency of blood flow velocity in the middle cerebral artery on end-tidal carbon dioxide partial pressure-a transcranial ultrasound Doppler study. J Cereb Blood Flow Metab. 1984:4:368-72.

17. Mccarville MB. Comparison of duplex and nonduplex transcranial Doppler ultrasonography. Ultrasound Q. 2008;24:167-71.

18. Mcmahon CJ, Mcdermott P, Horsfall D, et al. The reproducibility of transcranial Doppler middle cerebral artery velocity measurements: implications for clinical practice. Br J Neurosurg. 2007;21:21-7.

19. Mirzai H, Tekin I, Tarhan S, et al. Effect of propofol and clonidine on cerebral blood flow velocity and carbon dioxide reactivity in the middle cerebral artery. J Neurosurg Anesthesiol. 2004;16:1-5.

20. Muizelaar JP, Marmarou A, Ward JD, et al. Adverse effects of prolonged hyperventilation in patients with severe head injury: a randomized clinical trial. J Neurosurg. 1991;75:731-9.

21. Newell DW, Aaslid R, Stooss R, et al. Evaluation of hemodynamic responses in head injury patients with transcranial Doppler monitoring. Acta Neurochir (Wien). 1997:139:804-17.

22. Newell DW, Weber JP, Watson R, et al. Effect of transient moderate hyperventilation on dynamic cerebral autoregulation after severe head injury. Neurosurgery. 1996;39:35-43 discussion 43-34.

23. Nordstrom $\mathrm{CH}$, Messeter $\mathrm{K}$, Sundbarg G, et al. Cerebral blood flow, vasoreactivity, and oxygen consumption during barbiturate therapy in severe traumatic brain lesions. J Neurosurg. 1988;68:424-31.

24. Overgaard J, Tweed WA. Cerebral circulation after head injury. 1. Cerebral blood flow and its regulation after closed head injury with emphasis on clinical correlations. J Neurosurg. 1974;41:531-41.

25. Paulson OB, Strandgaard S, Edvinsson L. Cerebral Autoregulation. Cerebrovasc Brain Metab Rev. 1990:2:161-92.

26. Rangel-Castilla L, Lara LR, Gopinath S, et al. Cerebral hemodynamic effects of acute hyperoxia and hyperventilation after severe traumatic brain injury. J Neurotrauma. 2010;27:1853-63.

27. Sakai K, Cho S, Fukusaki M, et al. The effects of propofol with and without ketamine on human cerebral blood flow velocity and $\mathrm{CO}(2)$ response. Anesth Analg. 2000;90:377-82.

28. Strebel S, Kaufmann M, Guardiola PM, et al. Cerebral vasomotor responsiveness to carbon dioxide is preserved during propofol and midazolam anesthesia in humans. Anesth Analg. 1994;78:884-8.

29. Widder B, Paulat K, Hackspacher J, et al. Transcranial Doppler CO2 test for the detection of hemodynamically critical carotid artery stenoses and occlusions. Eur Arch Psychiatry Neurol Sci. 1986;236:162-8.

30. Zeiler FA, Ercole A, Placek MM, et al. Association between Physiological Signal Complexity and Outcomes in Moderate and Severe Traumatic Brain Injury: A CENTER-TBI Exploratory Analysis of Multi-Scale Entropy. J Neurotrauma. 2021;38(2):272-82.

\section{Publisher's Note}

Springer Nature remains neutral with regard to jurisdictional claims in published maps and institutional affiliations. 